\title{
SISTEMA DE RASTREAMENTO PARA PAINÉIS SOLARES NA CAPTAÇÃO DE ENERGIA
}

\author{
Bruno Foureaux Fabbis - bruno.fabbis@alunos.unis.edu.br \\ Estevão de Oliveira Conegundes - estevão.oc@gmail.com.br \\ Murilo Almeida Costa- Murilo - almeida30@hotmail.com \\ Vitor Martins Vale Menezes - vitorvmenezes@outlook.com \\ Zarur Silvano - zarurs@yahoo.com.br
}

\begin{abstract}
RESUMO: Este trabalho tem como objetivo o desenvolvimento de um Sistema de Rastreamento Solar aplicado a um painel com uso de sensores (LDR). Foi proposto um mecanismo de rastreamento junto ao painel solar, visando o acompanhamento do feixe de luz. Para avaliação desse sistema foi envolvido equipamentos de pequenas dimensões (escala reduzida) e equipamentos eletrônicos de baixa potência, facilitando assim o trabalho em bancada, para montagem e calibração. Com os resultando, verificou-se que o desenvolvimento com placa controladora Arduino possui várias aplicações reais e de ampla utilização. O funcionamento foi convincente, a planta se comportou bem aos comandos do Arduino. Ao final do trabalho foi realizada uma análise de proporção, evidenciando a utilização dos sensores (LRD), foram realizados testes com lanterna e luz ambiente, alcançando respectivamente aos feixes de luz.
\end{abstract}

Palavras-chave: LDR Automação. Arduino. Microcontrolador.

\section{INTRODUÇÃO}

O sol é a estrela central do universo e através de sua energia,na forma de radiação, é possível a vida na Terra. Vivemos em um mundo onde existe uma grande demanda por energia elétrica e como consequência energias renováveis. As fontes de energia renovável ganham espaço, pois, a energia proveniente dos combustíveis fósseis vai acabar em algum momento, além de ser uma fonte energética poluente.

Uma das formas mais utilizadas para conversão de energia solar em energia elétrica é através de células fotovoltaicas. Contudo, um painel estático possui limites na captação de energia solar pelo fato de sua imobilidade. Visando isso, pensou-se em um painel que se movesse em direção à fonte com maior intensidade de luz, gerando assim mais energia

O objetivo deste trabalho é mostrar que é possível que a placa fotovoltaica movase, direcionando-se à fonte de maior intensidade de luz permanecendo com toda placa iluminada. 


\section{METODOLOGIA}

De acordo Tardin et al (2013) apud Gil (1999), a metodologia é uma série de raciocínio. Foram realizadas pesquisas bibliográficas por meio de livros e internet, também foram consultados artigos científicos. Toda a elaboração do relatório técnico e construção do Protótipo foram realizadas entre o período de Setembro a Novembro de 2018. Toda coleta de dados para a estruturação do presente artigo ocorreramno período, entre 03 de Março a 31 de Maio de 2019. Para a execução do trabalho, foi feita inicialmente uma pesquisa bibliográfica, com o intuito de buscar referências de otimização na captação de luz.

O protótipo foi separado em duas etapas: sendo que a primeira o desenvolvimento da montagem eletrônica e a segunda do algoritmo. Segue Diagrama 1 que apresenta a ilustração do protótipo.

Figura 1: Diagrama de distribuição

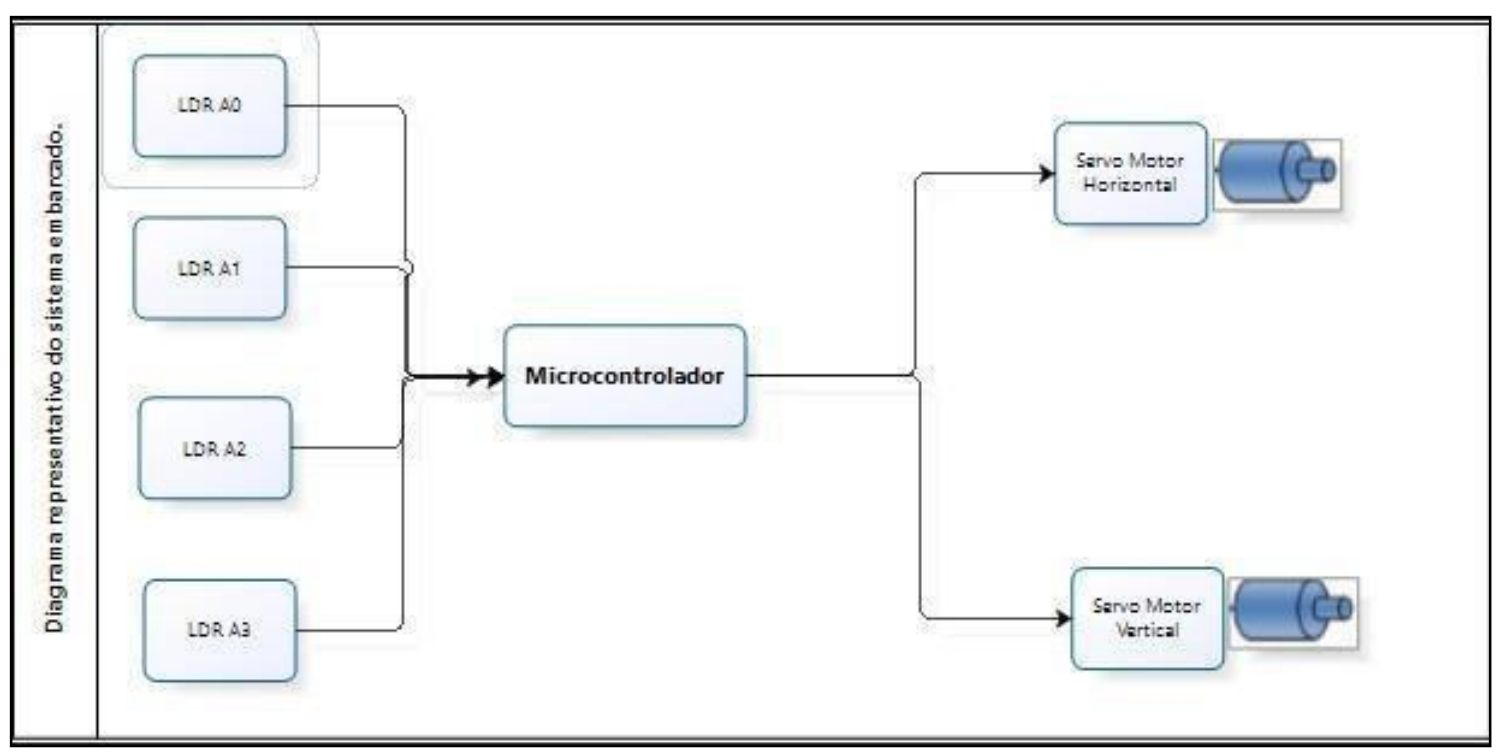

Fonte: Os Autores (2019)

Posteriormente, foi feita a elaboração do protótipo prático, sua construção física e integração entre suas diversas partes móveis, e o desenvolvimento de seu software embarcado apoiado na Lógica $\mathrm{C}++$. Toda a programação do Software Arduino segue em ApêndiceI, a este artigo. Para elaboração do circuito, visando um melhor entendimento e 
apoio na montagem, foi utilizado o software fritzing. Segue abaixo Figura 2, que representam circuito elaborado.

Figura 2: Circuito

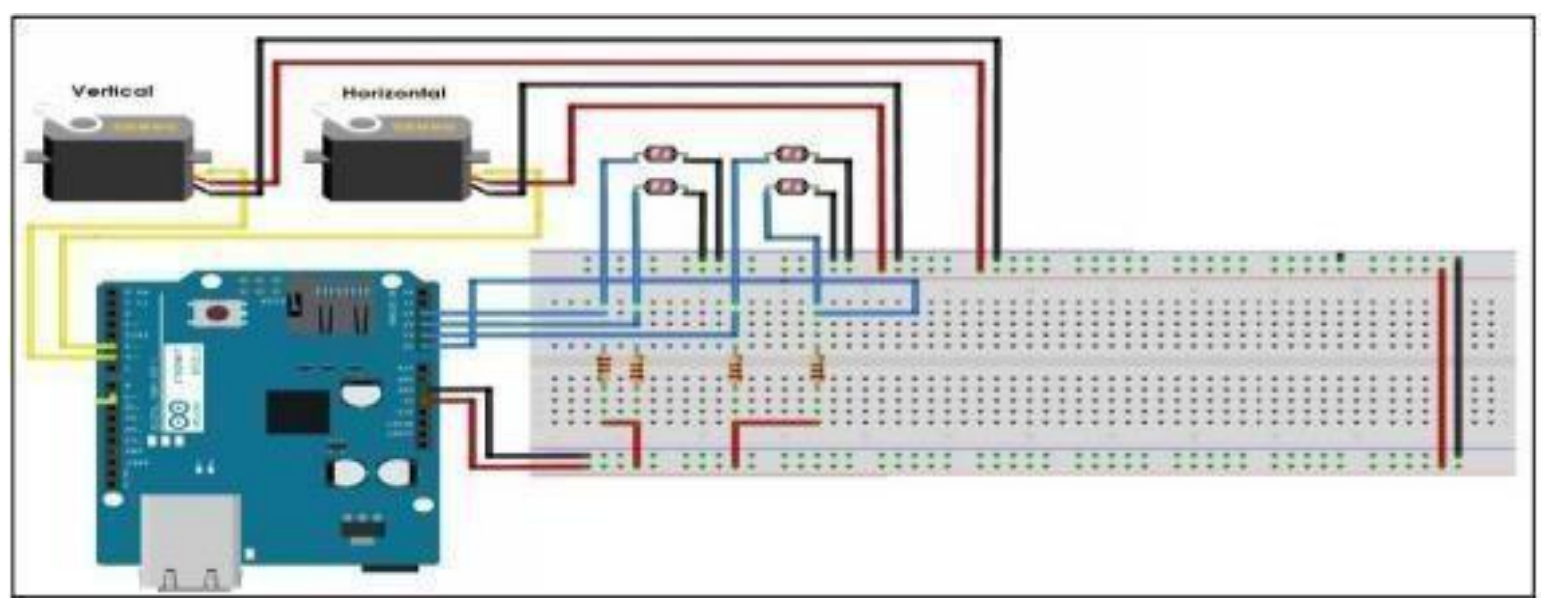

Fonte: Os Autores (2019)

Para a validação do modelo experimental, foram executados diversos testes práticos com lanterna e também ao ar livre, para fins de visualização do acompanhamento do painel em todo ciclo de rotação da maior intensidade de luz.

\section{FUNDAMENTAÇÃO TEÓRICA}

\subsection{Radiação Solar}

Segundo Fontes et al (2014) apud CRESESB (1999) o aproveitamento mais utilizado para conversão da energia solar em elétrica é a partir das células fotovoltaicas. Isso por ser uma energia infinita, limpa e silenciosa. Uma problemática quanto a seu uso é a baixa eficiência na conversão da energia, no máximo $30 \%$ dos raios solares são transformados em energia elétrica. Uma forma de melhorar o aproveitamento da radiação solar deve ser ajustando à posição do painel solar de acordo com a latitude local e o período do ano em que se requer mais energia. No Hemisfério Sul, por exemplo, um sistema de captação solar fixo deve ser orientado para o Norte, com ângulo de inclinação similar ao da latitude local. Já na Região Nordeste que apresenta uma cor laranja (Figura

3) tem uma insolação média anual alta, sendo assim, viável a geração de energia solar. 
Figura 3: Mapa Solarimétrico do Brasil, parte Região Nordeste

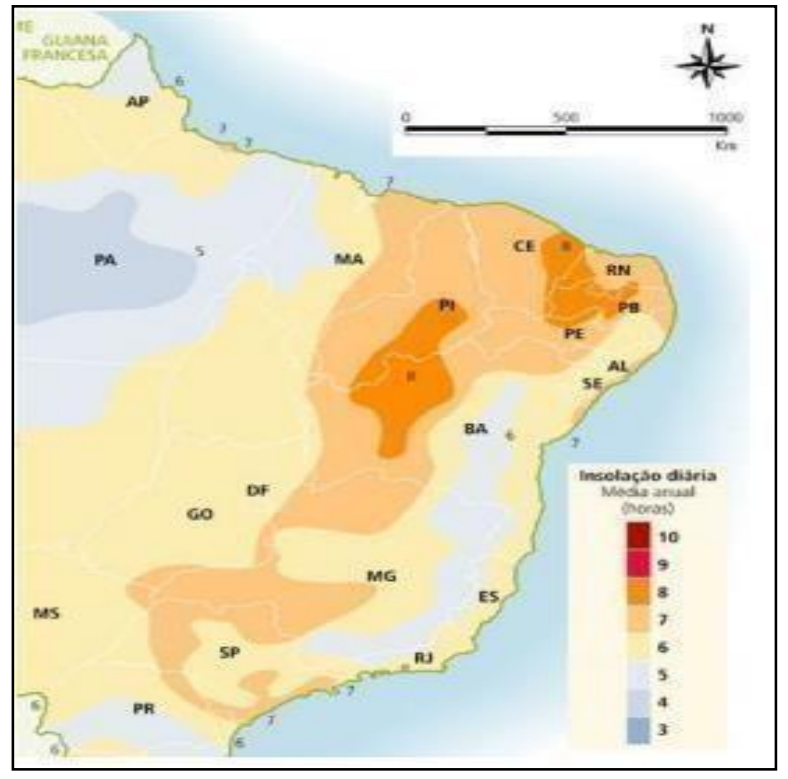

Fonte: Cresesb (2014)

Segundo Medeiros et al(2014 apud CRESESB, (1999), na Região Nordeste, que aponta uma cor laranja existe insolação média anual elevada, desta forma, acessível a geração de energia solar. A radiação solar atinge a superfície terrestre, necessário à reflexão e absorção dos raios solares pela atmosfera. Estima-se que a energia solar incidente sobre a superfície terrestre seja da ordem de 10 mil vezes o consumo energético Mundial.

Segundo Lima et al (2014) apud CRESESB, (1999) a Atlas Solarimétricodo Brasil (2000) mostra uma aferição da radiação solar incidente no país, consequente da interpolação e extrapolação de dados alcançados em estações solarimétricas separadas em vários pontos do território nacional. Apropriado, porém, ao número moderadamente reduzido de estações práticas e às variações climáticas locais e regionais, a Atlas de Irradiação Solar no Brasil faz estimativas de feixe solar a partir de expressões de satélites. Como pode ser validado, os supremos índices de radiação são observados na região Nordeste, com maior foco para o Vale do São Francisco. Uma forma de conseguir eficiência na captação de energia solar é através do movimento das placas, acompanhando o feixe de luz em todo seu ciclo. 


\subsection{Movimento de Rastreamento Solar}

Segundo Alcântara (2008), o dispositivo empregado para rastreamento do sentido do painel, concentra a energia de forma que o painel esteja sempre voltado para o raio solar, para que haja uma maior incidência de raios perpendiculares a superfície do mesmo. A eficiência do sistema aumenta com a maior incidência dos raios solares são considerados paramentos para o deslocamento: declividade terrestre, latitude, ângulo horário e dia do ano.Na maioria dos aplicativos de sistema ativos de seguimento,os sensores da placa de sombreamento são suficientes para determinar a posição solar através do sistema eletrônico. Com o emprego do LDR foi possível captar qualquer feixe de luz e tratar as informações obtidas com lógicas de comparações entre eles.

\subsection{Fotoresistor (LDR)}

De acordo com Wendling (2010) os LDR contêm uma face de sulfeto de cádmio (Cds) que tem sua resistência elétrica submetido da quantidade de luz incidente. O mesmo autor também relata da vantagem do uso do LDR como sensor fotoelétricos, destacando o fato de que eles ordem trabalhar com correntes relativamente altas, sendo assim muito sensíveis, o que simplifica o projeto de seus circuitos. Segue abaixo figura 4, ilustrando o LDR utilizado:

Figura 4: LDR Resistor dependente de luz

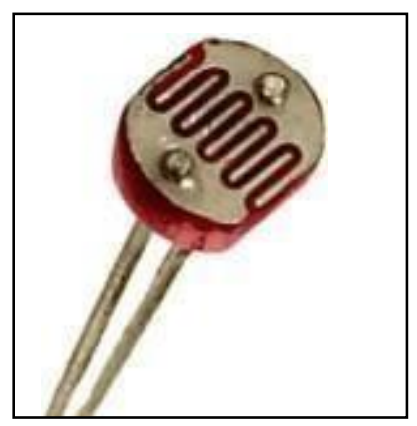

Fonte: Wendling (2010)

No presente trabalho os LDR assumiram a função de captar luz e realizar uma comparação entre os 4 , de forma que os mesmos busque a maior intensidades de luz, toda a 
lógica do programa foi realizada no microcontrolador Arduino.

\subsection{Microcontrolador}

Lana (2018) afirma que Arduino e uma plataforma de Hardware livre que possui um microcontrolador Atmel AVR de 8 bits, sendo possível programa-lo em $\mathrm{C} / \mathrm{C}++$. $\mathrm{O}$ Arduino é amplamente utilizado para fazer protótipos,robótica, automação e aprendizado de eletrônica devido a facilidade de uso e custo.

De acordo com Hagan e Culkin (2018) Arduino é um computador simples, de pequena escala e com preço acessível que se concentra na interação como mundo externo, ou seja, permite obter informações do mundo externo com sensores que medem temperatura, níveis de luz e de som, ou mesmo as vibrações sobre os pés, convertendo essa medida em movimento, som, luz e muito mais. Abaixo segue figura 5, referente a placa utilizada no protótipo

Figura 5: Placa de Microcontrolador - Arduino

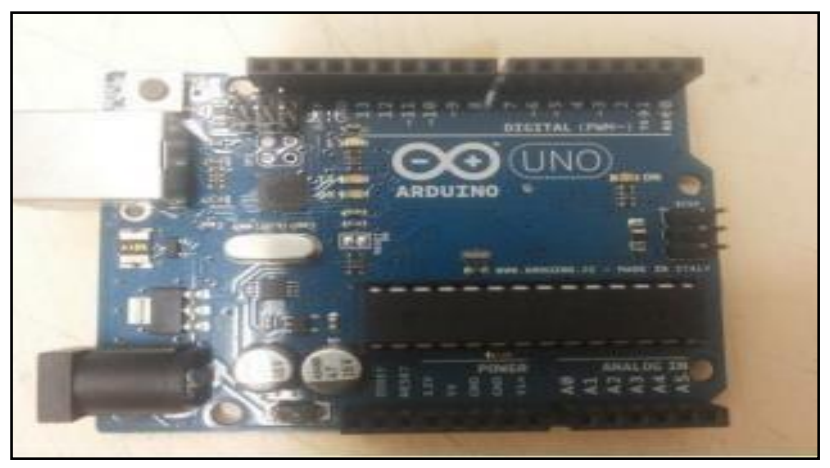

Fonte: Os Autores (2019)

O microcontrolador Arduino parte do conceito de hardware e software livre e está aberto para uso e contribuição de toda sociedade. A versão do hardware utilizado no Projeto é o Arduino Uno. Esta conta com 14 Pinos E/S digitais podem ser usados como saídas; 6 pinos entrada analógica e memória flash $32 \mathrm{~kb}$ utilizadas no projeto

O Arduino também é responsável pelo controle do servo motor nas direções horizontais e verticais, que é uma máquina de sistema eletromecânico para controle de rastreamento que executa um movimento de acordo com o comando específico, verificando 
sua atual posição pelo sol e chegando até o local desejado, diferentemente dos servomotores de rotação contínua.

\subsection{Servomotor}

Na concepção do autor, o servo motor é um pequeno motor DC acoplado através de um sistema de engrenagens a uma carga, sobre a qual fizemos o controle da posição. MAITELLI (1999). O servo utilizado apresenta um comprimento de 32,2mm, largura de $11,6 \mathrm{~mm}$ e altura de $31 \mathrm{~mm}$. A figura 6 ilustra o servo motor vertical e horizontal para proporcionar a redução da velocidade: $0,12 \mathrm{seg} / 60 \mathrm{Graus}$, a fim de fornecer um torque suficiente para a aplicação. É similar ao motor vertical e sua rotação de torque 4,8V.

Figura 6: Micro Servo 9g SG90

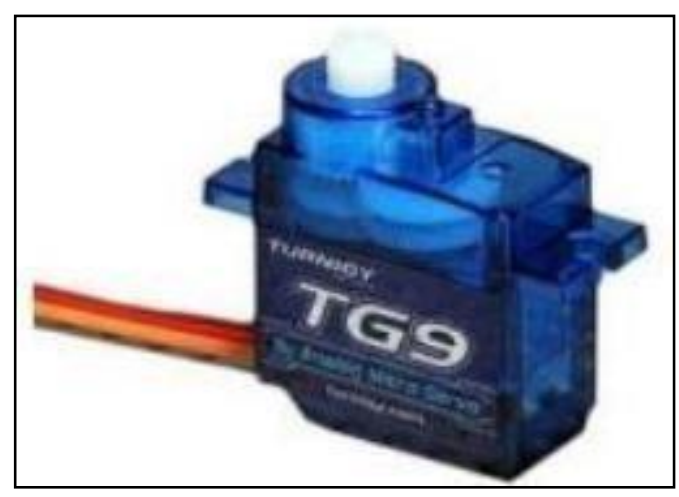

Fonte: Os Autores (2019)

Após os componentes adquiridos e posicionados em seus devidos lugares, tivemos um resultado satisfatório, resultando a uma sustentável e com todos os componentes bem destruídos em suas devidas funções.

\section{RESULTADOS E DISCUSSÕES}

A Figura 7 ilustra o dispositivo de bancada desenvolvido, os principais componentes utilizados (LDRs, Arduino e servomotor). Para proporcionar sustentação ao sistema usa- se: uma caixa de MDF (Madeira de Fundo) e cavilha para a confecção dos eixos.Este material foi escolhido devido sua grande facilidade de manipulação para furos e montagens, necessitando apenas de furos e parafusos. O dispositivo apresentou alto nível de estabilidade 
suportando o peso do painel fotovoltaico. Contudo as dimensões finais são: comprimento de $250 \mathrm{~mm}$ e altura de $150 \mathrm{~mm}$.

Figura 7: Protótipo Montado

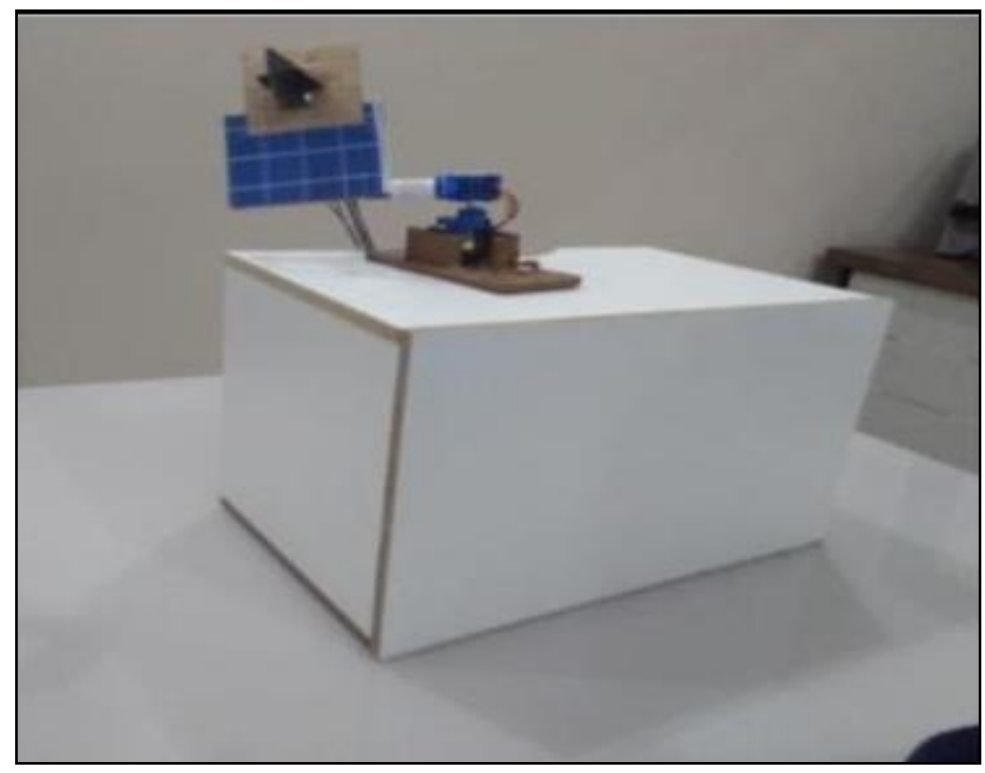

Fonte: Os Autores (2019)

A distribuição dos LDRs obedece uma certa lógica; estão da seguinte maneira: 2 LDRs identificados como superiores (direito e esquerdo), e dois inferiores (também direitoeesquerdo).Quando o bastão é endireitado para o sol ou o ponto mais brilhante os dados gerados pelos quatro LDR são enviados para o Arduíno onde serão tratados numa lógica que fará o protótipo se posicionar onde todos os LDR recebem a mesma quantidade de luz. Para ficar claro, foi considerado duassituações:

Situação 1: Luz na parte superior.

A planta proporciona sombra na parte inferior, e os servos atuarão de forma a elevar à base onde estão dispostos os LDR que simula o funcionamento da futura placa Solar.

Situação 2: Luz na parte inferior.

A planta proporciona sombra na parte superior, e os servos atuarão de forma a abaixar a base onde estão dispostos os LDRs que simula o funcionamento da futura placa solar.

A Lógica é mesma para qualquer possível situação.

Como resultado atingiu-se o controle de posição em torno dos dois eixos, mas, pela 
limitação do próprio equipamento atuador (servo) em girar apenas $180^{\circ}$,e também pelo fato de não ser os melhores LDR disponíveis no mercado, alguns movimentos da planta ficaram limitados, apesar de saber em qual posição se captura a maior incidência de luz, contudo, foram poucas as limitações. Fora isso, o funcionamento foi convincente e a planta se comportou bem aos comandos do Arduino.

\section{CONCLUSÃO}

Com base no estudo e na montagem realizada foi possível montar os componentes na devida placa, e o suporte que irá movimentar os LDR e desenvolver o algoritmo que permita que os LDR se movimente de acordo com o ponto de captação de maior incidência de luz. Verificou-se que o desenvolvimento com placa controladora Arduino possui várias aplicações reais e de ampla utilização. Foi possível revisar conhecimentos interdisciplinares, agregando a ementa tradicional com as disciplinas técnicas do curso, aumentando assim a assimilação dos conceitos e desenvolvimento prático.

Todo o sistema apresenta similaridades ao processo real e, por isso, é algo promissorno âmbito da automação. Portanto, deum modo geral, vão existir muitos gargalos na aplicação do protótipo em uma projeto real, passando a abrir um leque de possibilidades para a automação, gerando um grande desafio em uma aplicação de grande escala. $\mathrm{O}$ funcionamento foi convincente, a planta se comportou bem aos comandos do Arduino e, por isso, pretendemos dar continuidade, visando um estudo relacionado à aplicação com equipamentos de grande escala do ramo de energia solar.

\section{REFERÊNCIA BIBLIOGRÁFICA}

ALCÂNTARA, S. M. F (2008). Análise do Desempenho de um Painel Fotovoltaico de Baixa Tensão Acoplado a um Conversor DC-DC: Topologia Booster. Disponível em: http://www.abcm.org.br/pt/wpontent/anais/conem/2010/PDF/CON10-1655.pdf . Acesso em: 08 de Maio de 2019.

FONTES ET AL (2014) APUD CRESESB (1999)UseofAutomated Systems toOptimize Energy capture in solar panels. Disponível em: http://pdf.blucher.com.br.s3-sa-east-

1.amazonaws.com/mathematicalproceedings/cnmai2014/0006.pdf. Acesso em: 24 de Maio de2019.
HAGAN
e
CULKIN
(2018). AprendaEletrônica
com
Arduino.
Disponível

https://books.google.com.br/books?id=fxZSDwAAQBAJ\&amp;printsec=frontcover\&amp;dq=arduino\&a $\mathrm{mp}$;hl=pt-BR\&amp;sa\#v=onepage\&amp;q=arduino\&amp;f=false. Acesso em 05 de Maio de2019.

KALOGIROU (2017). Engenharia de Energia Solar. Disponível em: https://books.google.com.br/books?id=_q44DwAAQBAJ\&amp;printsec=frontcover\&amp;dq=energia+so 
lar\&amp;hl=pt-BR\&amp;sa=X\&amp;ved=0ahUKEwinvM

QnsHiAhWUHrkGHTeID18Q6AEILTAB\#v=onepage\&amp;q\&amp;f=false. Acesso em 01 de Maio de 2019.

LANA (2018). Projetos Maker. Disponível em: https://books.google.com.br/books?id=xh50DwAAQBAJ\&amp;printsec=frontcover\&amp;dq=arduino\&amp; hl=ptBR\&amp;sa=X\&amp;ved=0ahUKEwjL6eju8cDiAhWPCrkGHWOcCHU4ChDoAQheMAk\#v= onepage\&amp;q=arduino\&amp;f=false. Acesso em: 04 de Maio de2019.

LANA (2018). Projetos Maker. Disponível em: https://books.google.com.br/books?id=xh50DwAAQBAJ\&amp;printsec=frontcover\&amp;dq=arduino\&amp; hl=ptBR\&amp;sa=X\&amp;ved=0ahUKEwjL6eju8cDiAhWPCrkGHWOcCHU4ChDoAQheMAk\#v= onepage\&amp;q=arduino\&amp;f=false. Acesso em: 04 de Maio de2019.

LIMA ET AL (2014) APUD CRESESB, (1999) Use ofAutomated Systems toOptimize Energy capture in solar panels. Disponível em: http://pdf.blucher.com.br.s3-sa-east-

1.amazonaws.com/mathematicalproceedings/cnmai2014/0006.pdf. Acesso em: 24 de Maio de2019.

LÓCIO F (2010). Fluxograma 10/04/2010 Disponível em <http://www.scribd.com /doc/36162383/osm1fluxograma> Acesso em 24 de Maio de 2019.

MAITELLI (1999). Um Esquema de Neurocontrole com Treinamento em Tempo Real Aplicado ao Posicionamento de um Servomotor. Disponível em:

https://fei.edu.br/sbai/SBAI1999/ARTIGOS/IV_SBAI_12.pdf.Acessoem:24deMaiode2019.

MEDEIROS et al DANTAS (2014), apud CRESESB (1999). Use of Automated Systems to Optimize Energy capture in solar panels. Disponível em: http://pdf.blucher.com.br.s3-sa-east-

1.amazonaws.com/mathematicalproceedings/cnmai2014/0006.pdf. Acesso em: 24 de Maio de 2019.

OLIVEIRA, J. W. (2009) Sistema de Informação. Disponível em:

<https://xa.yimg.com/kq/groups/22755187/1481008806/name/Proc.Neg.Atividade.pdf. > Acesso em 24 de Maio de 2019.

REN21,(2011).renewables 2011 globalstatusreport.Disponívelem:

http://www.ren21.net/Portals/0/documents/Resources/GSR2011_FINAL.pdf.Acesso em: 01 de Maio de 2019.

TARDIN et al (2013) apud GIL (1999), XXXIII Encontro Nacional de Engenharia de Produção, Enegep. 2013 >Acesso em 24 de Maio de 2019.

WENDLING (2010), Sensores, Disponível em: https://paulocrgomes.com.br/wpcontent/uploads/2018/11/sensores.pdf.Acesso em 22 de Maio de2019

\section{APÊNDICE I - Código para programar a placa de Arduíno.}

\#include < Servo.h> // include Servo library Servo horizontal; // horizontal servo intservoh=0; // suporte horizontal

//

Servo vertical; // vertical servo intservov=0;// suporte vertical intldrlt = A1; //LDR superior esquerdo intldrrt = $\mathrm{A} 0 ; / / \mathrm{LDR}$ superiror direito intldrld = A3; //LDR inferior esquerdo intldrrd = A2; //LDR inferior direito void setup()

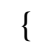

Serial.begin(250000);//250000 horizontal.attach(5); vertical.attach(6); \}

void loop() 
intlumSE = analogRead(ldrlt); // luminozidade superior esquerda intlumSD = analogRead(ldrrt); // luminozidade superior direita intlumIE $=$ analogRead(ldrld); // luminozidade inferior esquerda intlumID $=$ analogRead(ldrrd); // luminozidade inferior direita inttolerancia $=40$; int SSS = (lumSE + lumSD) / 2; // media das leituras superiores int III = (lumIE + lumID) / 2; // media das leituras inferiores

int SIE = (lumSE + lumIE) / 2; // media das leituras superior e inferior esquerdo int SID = (lumSD + lumID) / 2;// media das leituras superior e inferior direito interroVertical = SSS - III; // base para atuação do servo davertical interroHorizontal $=$ SIE - SID; // base para a atuação do servo da horizontal if (abs(erroVertical) $>$ tolerancia) \{

if $(\mathrm{SSS}>\mathrm{III})\{$

servov $=$

++ servov; if (servov> 180) \{

servov $=180$;

\}

\}

else if (SSS < III)

$\{$ servov $=--$ servov; if

$($ servov< 0$)\{$

servov $=0$;

\}

\}

vertical.write(servov);

\}

if (abs(erroHorizontal) >tolerancia) // Verifica se a diferença naoesta dentro da toleranciaerancia definida \{

//if (SIE

$>$ SID) if (SIE

$<$ SID)

\{

servoh $=-$-servoh; if $($ servoh $<0)\{$ servoh $=0 ;$

\}

\} 
ISSN 1994-0098

else if (SIE $>$ SID) $\{/ /($ SIE $<$ SID $)\{$

servoh $=$

++ servoh; if (servoh> 180) \{

servoh $=180$;

\}

\}

else if $($ SIE $==$ SID $)\{$

// nothing to do

\}

horizontal.write(servoh); delay(20);

\}

//Data enviada para o sistema supervisório Serial.print("Estado dos sensores:");

Serial.print("

Serial.print(" LDR conectado na porta A0: "); Serial.print( $\operatorname{analogRead(A0));~");~}$

Serial.print(" ");

Serial.print(" LDR conectado na porta A1: ");

Serial.print(analogRead(A1));

Serial.print(" ");

Serial.print(" LDR conectado na porta A2: ");

Serial.print(analogRead(A2));

Serial.print(" ");

Serial.print(" LDR conectado na porta A3: ");

Serial.print(analogRead(A3));

Serial.print(" ");

Serial.print("Estado dos Atuadores:");

Serial.print(" ");

Serial.print(" Angulação do servo da Horizontal: ");

Serial.print(servoh);

Serial.print(" ");

Serial.print(" Angulação do servo da Vertical: ");

Serial.println(servov); 